\title{
PRIMER REGISTRO DEL GÉNERO CABAMOFA JASCHHOF PARA MÉXICO (DIPTERA: BIBIONOMORPHA)
}

\author{
Herón HUERTA ${ }^{1} \&$ FELIPE DZUL ${ }^{2}$
}

\author{
${ }^{1}$ Laboratorio Entomología, Instituto de Diagnóstico y Referencia Epidemiológicos-InDRE, \\ Calle Francisco de P. Miranda No. 177, Col. Unidad Lomas de Plateros, Del. Álvaro Obregón, 01480, México, D. F. \\ <cerato_2000@yahoo.com> \\ ${ }^{2}$ Departamento de Prevención y Control de Enfermedades Transmitidas por Vector, \\ Servicios Estatales de Salud de Guerrero, Av. Ruffo Figueroa No.6, Col. Burócratas, \\ 39090, Chilpancingo, Guerrero, México <fdzul@me.com>
}

Recibido: 05/12/2014; aceptado: 18/01/2016

Huerta, H. \& Dzul, F. 2016. Primer registro del género Cabamofa Jaschhof para México (Diptera: Bibionomorpha). Acta Zoológica Mexicana (n. s.), 32(1): 1-5.

RESUMEN. Se presenta el primer registro del género Cabamofa Jaschhof para México, con base en cuatro especímenes hembras procedentes de la localidad de Acahuizotla, Guerrero. Los especímenes fueron recolectados mediante trampas de luz tipo CDC miniatura, los cuales se describen, ilustran y se comparan con Cabamofa mira Jaschhof.

Palabras clave: Grupo Ohakunea, Rangomaramidae, nuevo registro.

\section{INTRODUCCIÓN}

El género Cabamofa Jaschhof fue establecido para la única especie conocida de Costa Rica, Cabamofa mira Jaschhof (2005), con base en la descripción de dos ejemplares hembra procedentes del Parque Nacional de Corcovado, Costa Rica. Posteriormente, Amorim \& Rindal (2007) describen el macho conespecífico, con base en un ejemplar colectado de Turrialba, Costa Rica. De acuerdo con el reciente catálogo Neotropical de la familia Rangomaramidae Jaschhof \& Didham, 2002, ésta familia incluye seis géneros y 20 especies (Amorim \& Falaschi 2012). El género Cabamofa Jaschhof (2005) se agrupa dentro de la subfamilia Ohakuneinae Amorim \& Rindal (2007), la cual incluye otros tres géneros relacionados entre sí, Ohakunea Tonnoir \& Edwards (1927), Colonomyia Colles (1963) y Rogambara Jaschhof (2005). La actual controversia por la posición taxonómica de la subfamilia Ohakuneinae (Amorim \& Rindal 2007) o grupo Ohakunea (Jaschhof 2005, 2009) ha llevado a diferentes hipótesis sobre las relaciones filogenéticas del grupo (Jaschhof \& Hippa 2003), por lo que dicho taxa es considerado en la actualidad como grupo Ohakunea, sin una asignación particular a alguna familia (Jaschhof 2009). Este peculiar
Huerta, H. \& Dzul, F. 2016. First record of the genus Cabamofa Jaschhof for Mexico (Diptera: Bibionomorpha). Acta Zoológica Mexicana (n. s.), 32(1): 1-5.

ABSTRACT. The first record of the genus Cabamofa Jaschhof from Mexico is presented, based in four females from the locality of Acahuizotla, Guerrero. Specimens were collected by miniature CDC light traps, which are described, illustrated, and compared with Cabamofa mira Jaschhof.

Key words: Ohakunea group, Rangomaramidae, new record.

género se caracteriza principalmente por la distintiva venación alar $\left(\mathrm{R}_{2+3} \mathrm{y}\right.$ base de la $\mathrm{M}$ ausente), ocelos ausentes, ojos cercanos entre sí por arriba de la inserción antenal, palpo con cinco segmentos y patas con la tibia media y la posterior con una espina apical.

En el muestreo llevado a cabo cerca de la localidad de Acahuizotla, Guerrero, se capturaron cuatro ejemplares hembra del género Cabamofa Jaschhof. Por lo tanto, el objetivo del presente trabajo es dar a conocer el primer registro de este género para México y compararlos con la otra especie conocida, Cabamofa mira Jaschhof (2005).

\section{MATERIAL Y MÉTODOS}

Los ejemplares fueron capturados mediante trampa de luz CDC miniatura y posteriormente preservados en etanol al $70 \%$ para su estudio. Tres hembras fueron preparadas en laminillas para su estudio microscópico. Los ejemplares fueron macerados con hidróxido de potasio al 8\% en baño María, lavados en agua destilada caliente, ácido acético al 10\%, alcohol isopropílico mezclado con aceite de clavo, aceite de clavo puro y finalmente incluidos en resina Euparal. Los términos morfológicos y de nomenclatura 
empleados fueron basados en Amorim \& Rindal (2007) y Jaschhof (2005). El material estudiado se encuentra depositado en la Colección de Artrópodos con Importancia Médica (CAIM), del Instituto de Diagnóstico y Referencia Epidemiológicos, (InDRE), en la Ciudad de México. Las medidas se proporcionan en milímetros. Algunas proporciones se integran en la descripción, indicando el valor promedio y entre paréntesis el rango y el número de mediciones. Las ilustraciones fueron realizadas mediante cámara clara de dibujo y posteriormente trazadas en tinta. Las fotografías se editaron con el uso del programa Adobe Photoshop.

\section{RESULTADOS}

\section{Cabamofa sp.}

(Figuras 1A-J, 2A-D)

Material Examinado. 4 hembras (CAIM). MÉXICO, Guerrero, Municipio Chilpancingo, Localidad Acahuizo-

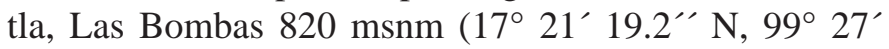
12.8” W), 5-Noviembre-2010, trampa de luz tipo CDC miniatura, Colectores Dzul, F., Bibiano Marín, W. Tres hembras preservadas en laminillas, una hembra preservada en alcohol etílico al 70\%.

Descripción. Hembra. Longitud total $3.1 \mathrm{~mm}$. Coloración. Cabeza pardo oscuro, incluyendo el tubérculo frontal, frente, vértice y parte dorsal del occipucio; antena con escapo pardo oscuro, pedicelo y flagelómeros pardo claro; partes bucales pardo claro. Tórax, patas y halterios pardo claro; ala translúcida; terga y esterna abdominales pardos, excepto el primer esternón que es pardo oscuro.

Cabeza (Fig. 1A). Puente ocular separado por una distancia igual al diámetro de 3 a 4 facetas; sedas interfacetarias presentes; escapo cilíndrico, con sedas presentes únicamente en la parte ventro-lateral; pedicelo cilíndrico, similar en longitud al escapo, con sedas dispuestas en la parte distal; antena $3.1 \mathrm{x}$ veces más larga que la anchura de la cabeza, longitud antenal (Figs. 1B, D) 1.25 mm (1.20$1.30 \mathrm{~mm}, n=3$ ) con 14 flagelómeros, cada uno formado por un nodo cilíndrico y con internodos cortos, cuarto flagelómero (Fig. 1C) 1.5x veces más largo que ancho; último flagelómero $0.125 \mathrm{~mm}(n=2)$, más largo que los flagelómeros precedentes; clípeo con un par de sedas latero-basales. Palpo (Figs. 1E-F), con el primer segmento presentando una seda latero-distal; segundo segmento cónico, más corto que el primer segmento, con un par de sedas latero-distales; tercer segmento globular, longitud $0.08 \mathrm{~mm}$, anchura $0.06 \mathrm{~mm}$; hueco sensorial profundo con abertura circular y con varias sedas distribuidas en todo el segmento; cuarto segmento casi cilíndrico, más ancho en su extremo distal, con sedas dispuestas desde la parte media hacia el ápice; quinto segmento largo, 0.125 $\mathrm{mm}(n=2), 2.5 x$ veces la longitud del cuarto segmento, con sedas laterales y tres sedas apicales.

Tórax (Fig. 1G). Escudo con abundantes sedas dispersas de diferentes longitudes; escutelo similar, pero con dos sedas largas que se originan de la parte central. Antepronoto con una seda sub-basal; episternón I con 8 sedas dispersas, margen adyacente al postpronoto con 3-4 sedas. Todos los demás escleritos sin sedas y con las mismas características descritas para Cabamofa mira Jaschhof (2005).

Patas (Figs. 1H-J). Coxas con sedas dispersas, en mayor abundancia en la coxa anterior; proporción de las patas: longitud fémur/tibia: I (0.90), II (0.85), III (0.80). Espolones tibiales 1:2:2. Tibia anterior con depresión anteroapical formado el peine tibial compuesto entre 8-10 sedas rígidas, un par de espinas apicales cortas (Fig. 1H); tibia media con 10 espinas apicales en peine (Fig. 1I), tibia posterior con peine subapical compuesto por 19-22 sedas rígidas (Fig. 1J); proporción tarsal (longitud del tarsómero 1/tarsómero 2): I (2.0), II (2.2), III (2.4). Tarsómeros 1 al 4 de todas las patas con hilera longitudinal de sedas dispuestas en empalizada y una espina apical ventral en los tarsómeros de la pata media y posterior; primer tarsómero de todas las patas con sedas gruesas adicionales.

Ala (Fig. 2A). Longitud $2.75 \mathrm{~mm}(2.70-2.80 \mathrm{~mm}, n=$ $3)$, anchura $1.05 \mathrm{~mm},(n=3)$. Venación con las mismas características descritas para Cabamofa mira Jaschhof (2005). Balancín con longitud de $0.11 \mathrm{~mm}$, sedas dispersas en el capítelo.

Abdomen. (Figs. 2B-C). Longitud $2.25 \mathrm{~mm}(n=2)$. Esternón 9 ligeramente más largo que ancho, con el margen posterior no marcadamente puntiagudo. Con dos espermatecas (Figs. 2B, D) similares en tamaño, piriformes, esclerotizadas con corto cuello; patrón lateral con apariencia moteada dispuesto cerca de la base del cuello; longitud $0.12 \mathrm{~mm}(n=2)$, anchura $0.11 \mathrm{~mm}(n=2)$.

Macho. Desconocido.

Distribución. México (Guerrero). Ejemplares capturados en Selva Baja Caducifolia (Fig. 3).

\section{COMENTARIOS TAXONÓMICOS}

La comparación de la descripción original de Cabamofa mira Jaschhof con los ejemplares de México, indica 

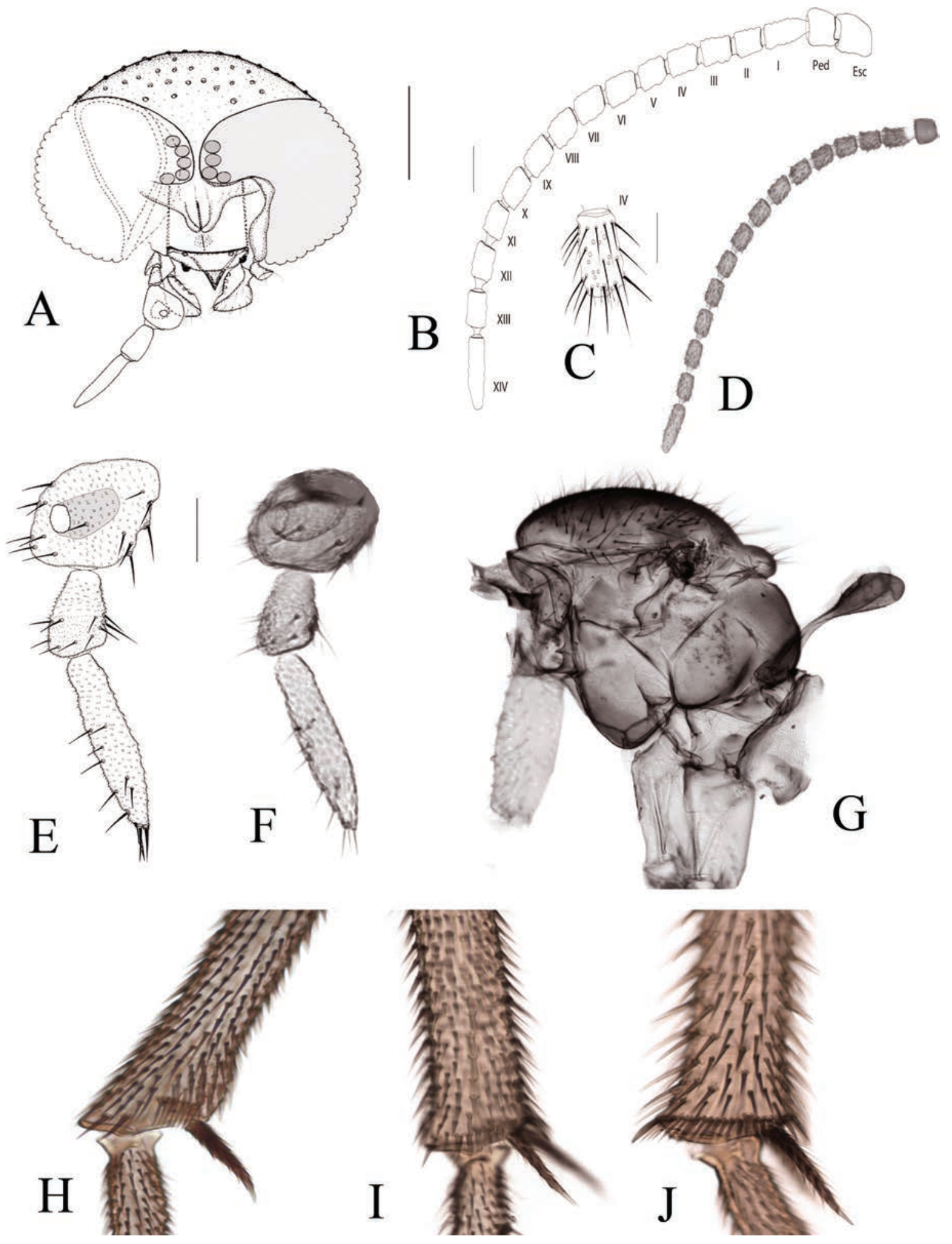

Figura 1. Hembras de Cabamofa sp. procedentes de México. (A) cabeza, vista frontal (escala = $0.16 \mathrm{~mm}$ ). (B, D) flagelo antenal, vista lateral $($ escala $=0.064 \mathrm{~mm}) .(\mathrm{C})$ cuarto flagelómero, vista lateral $($ escala $=0.04 \mathrm{~mm}) .(\mathrm{E}-\mathrm{F})$ palpo maxilar, segmentos 3-5 en vista lateral $($ escala $=$ $0.04 \mathrm{~mm}$ ). (G) tórax, vista lateral. (H-J) patas, parte apical de las tibias, vista lateral:(H)- tibia anterior, (I)- tibia media, (J)- tibia posterior. Abreviaturas: Esc = escapo; Ped = pedicelo; I-XIV = flagelómeros. 

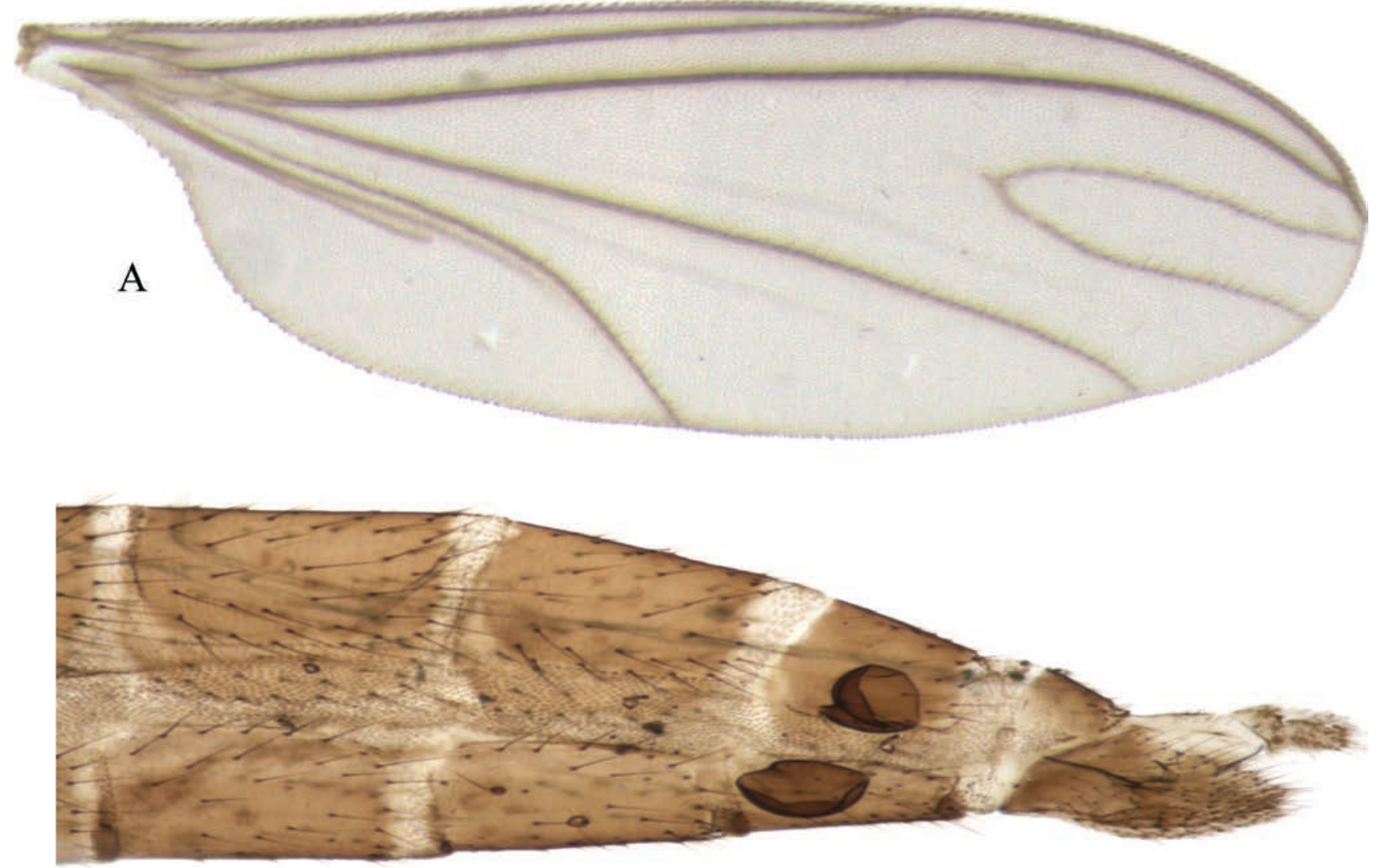

B
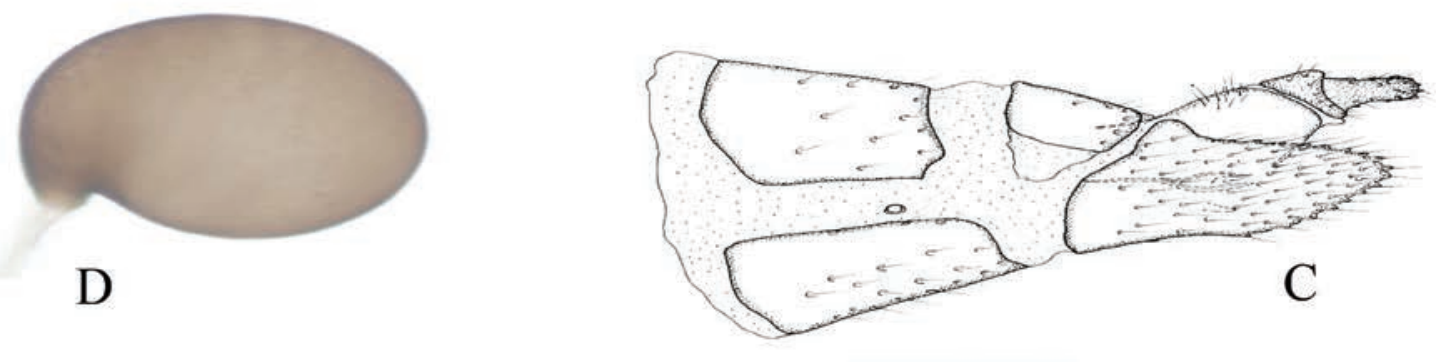

Figura 2. Hembras de Cabamofa sp. procedentes de México. (A) ala derecha, vista dorsal. (B-C) abdomen, últimos segmentos abdominales, vista lateral $($ escala $=0.16 \mathrm{~mm})$. $(\mathrm{D})$ espermateca, vista lateral.

que Cabamofa sp. parece ser conespecífica. Sin embargo, observamos algunas diferencias con respecto a la descripción de $C$. mira, en la cual se observan 5-6 facetas en línea vertical en el puente ocular; longitud del ala cercana a $2.0 \mathrm{~mm}$; segmentos del palpo cuarto y cinco similares en longitud; 12 sedas rígidas en el peine tibial de la pata anterior y 18 en la pata posterior; episternón I con 45 sedas; espermatecas en forma globular y esternón IX disminuyendo en anchura hacia el ápice, terminando con apariencia puntiaguda. Consideramos que las diferencias morfológicas descritas, podrían deberse a una variación intraespecífica de Cabamofa mira. Por lo tanto, en el presente estudio no es posible establecer la identidad plena de los especímenes mexicanos con base en las características de las hembras solamente, por lo que se requiere de especímenes machos, cuyas características de los terminalia son necesarias para distinguir éstas especies.

AGRADECIMIENTOS. Agradecemos a nuestro colega Wilbert Bibiano Marín, por su apoyo en el trabajo de campo y recolecta de los ejemplares. Así como también a los dos revisores por sus sugerencias y observaciones hechas al manuscrito. 


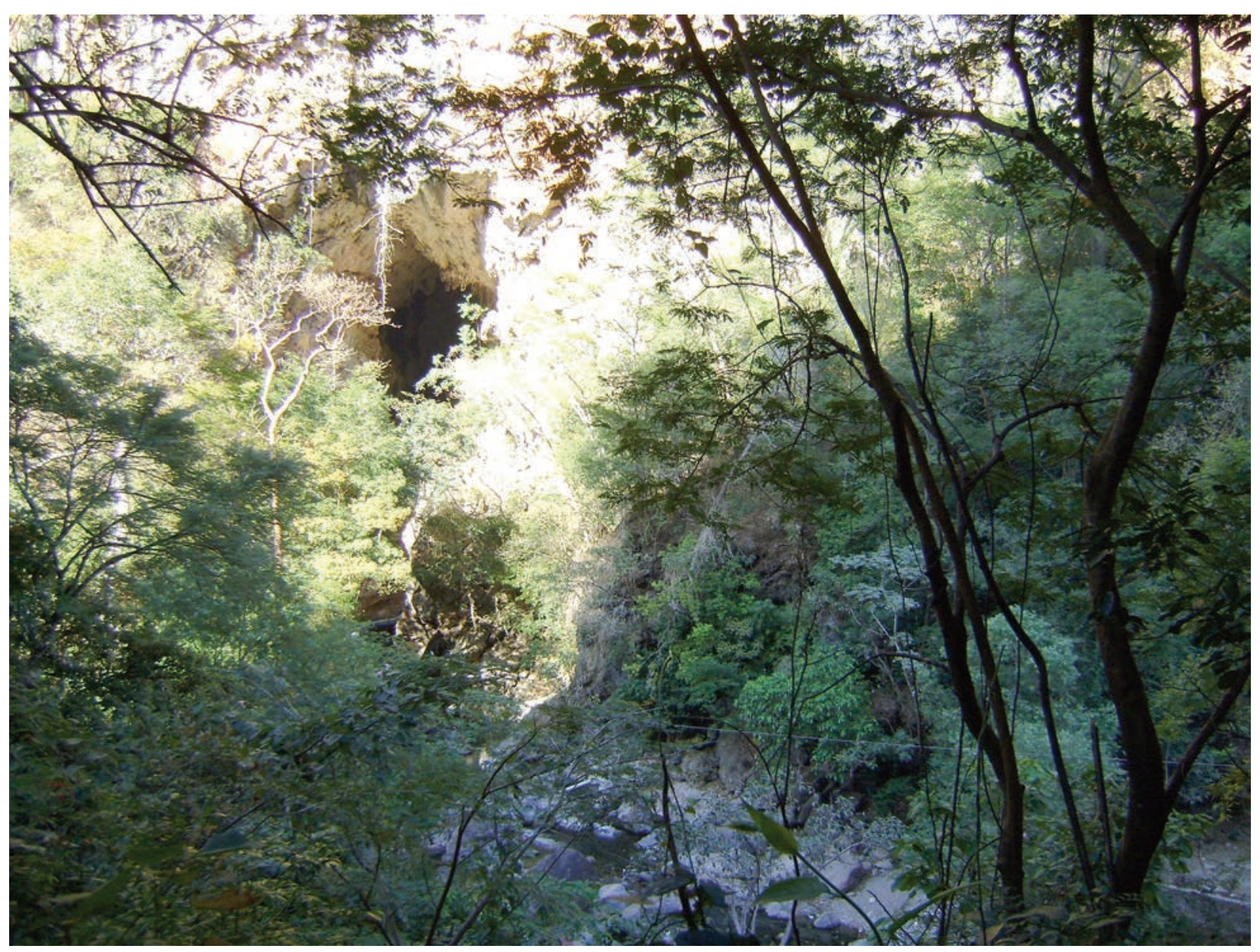

Figura 3. Hábitat característico de los especímenes estudiados en la localidad de Acahuizotla (Guerrero, México).

\section{LITERATURA CITADA}

Amorim, D. S. \& Rindal, E. 2007. Phylogeny of the Mycetophiliformia, with proposal of the subfamilies Heterotrichinae, Ohakuneinae, Chiletrichinae for the Rangomaramidae (Diptera: Bibionomorpha). Zootaxa, 1535: 1-92.

Amorim, D. S. \& Falaschi, L. R. 2012. Catalogue of Neotropical Diptera.Rangomaramidae. Neotropical Diptera, 21: 1-7.

Colless, D. H. 1963. New species of Ohakunea Edwards and related new genus with notes on the relationships of Heterotricha Loew (Diptera). Proceedings of the Linnean Society of New South Wales, 87: 303-308.

Jaschhof, M. (2004) 2005. Rogambara and Cabamofa, two new genera of enigmatic sciaroids from Costa Rica (Insecta: Diptera: Sciaroidea). Bonner zoologische Beiträge, 53: 323-332.
Jaschhof, M. 2009. Ohakunea group (Sciaroidea, unassigned to family; no common name), chapter 18, Pp. 315-317. In: B.V. Brown, A. Borkent, J.M. Cumming, D.M. Wood, N.E. Woodley, and M.A. Zumbado (Eds). Manual of Central American Diptera: Volume 1. NRC Research Press, Ottawa, Ontario, Canada.

Jaschhof, M. \& Didham, R. K. 2002. Rangomaramidae fam. nov. from New Zealand and implications for the phylogeny of the Sciaroidea (Diptera: Bibionomorpha). Studia dipterologica, Supplement 11: 1-60.

Jaschhof, M. \& Hippa, H. 2003. Sciaroid but no sciarid: a review of the genus Ohakunea Tonnoir \& Edwards, with the description of two new species (Insecta: Diptera: Bibionomorpha). Entomologische Abhandlungen, 60: 23-44.

Tonnoir, A. L. \& Edwards, F. W. 1927. New Zealand fungus gnats (Diptera, Mycetophilidae). Transactions of the New Zealand Institute, 57: 747-878. 